\title{
CT Image Conversion among Different Reconstruction Kernels without a Sinogram by Using a Convolutional
} Neural Network

\author{
Sang Min Lee, $M D^{1 *}$, June-Goo Lee, $\mathrm{PhD}^{2 *}$, Gaeun Lee, $B S^{2}$, Jooae Choe, $\mathrm{MD}^{1}$, Kyung-Hyun Do, $\mathrm{MD}^{1}$, \\ Namkug Kim, $\mathrm{PhD}^{1,2}$, Joon Beom Seo, $\mathrm{MD}^{1}$ \\ ${ }^{1}$ Department of Radiology and Research Institute of Radiology, University of Ulsan College of Medicine, Asan Medical Center, Seoul, Korea; \\ ${ }^{2}$ Department of Convergence Medicine, University of Ulsan College of Medicine, Asan Medical Center, Seoul, Korea
}

Objective: The aim of our study was to develop and validate a convolutional neural network (CNN) architecture to convert CT images reconstructed with one kernel to images with different reconstruction kernels without using a sinogram.

Materials and Methods: This retrospective study was approved by the Institutional Review Board. Ten chest CT scans were performed and reconstructed with the B10f, B30f, B50f, and B70f kernels. The dataset was divided into six, two, and two examinations for training, validation, and testing, respectively. We constructed a CNN architecture consisting of six convolutional layers, each with a $3 \times 3$ kernel with 64 filter banks. Quantitative performance was evaluated using root mean square error (RMSE) values. To validate clinical use, image conversion was conducted on 30 additional chest CT scans reconstructed with the B30f and B50f kernels. The influence of image conversion on emphysema quantification was assessed with Bland-Altman plots. Results: Our scheme rapidly generated conversion results at the rate of $0.065 \mathrm{~s} / \mathrm{slice}$. Substantial reduction in RMSE was observed in the converted images in comparison with the original images with different kernels (mean reduction, 65.7\%; range, 29.5-82.2\%). The mean emphysema indices for B30f, B50f, converted B30f, and converted B50f were 5.4 $\pm 7.2 \%$, $15.3 \pm 7.2 \%, 5.9 \pm 7.3 \%$, and $16.8 \pm 7.5 \%$, respectively. The $95 \%$ limits of agreement between B30f and other kernels (B50f and converted B30f) ranged from $-14.1 \%$ to $-2.6 \%$ (mean, $-8.3 \%$ ) and $-2.3 \%$ to $0.7 \%$ (mean, $-0.8 \%$ ), respectively. Conclusion: CNN-based CT kernel conversion shows adequate performance with high accuracy and speed, indicating its potential clinical use.

Keywords: Multidetector computed tomography; Image reconstruction; Machine learning; Emphysema; CNN

\section{INTRODUCTION}

Quantitative imaging in the medical field has been rapidly developing because of advances in computational methods and technologies. However, accuracy and reproducibility are essential for implementing quantitative imaging in clinical practice, and the technical parameters in imaging protocols should be carefully determined.

In CT, the reconstruction kernel is an important technical parameter, and kernel selection involves a trade-off between spatial resolution and image noise (1) because sharper kernels increase spatial resolution and image noise. Reconstruction kernel selection also depends on the structure being evaluated, e.g., while a sharp kernel is

Received April 19, 2018; accepted after revision August 7, 2018.

This study was supported funding by the Industrial Strategic technology development program (10072064, Development of Novel Artificial Intelligence Technologies To Assist Imaging Diagnosis of Pulmonary, Hepatic, and Cardiac Diseases and Their Integration into Commercial Clinical PACS Platforms) funded by the Ministry of Trade Industry and Energy (MI, Korea).

*These authors contributed equally to this work.

Corresponding author: Joon Beom Seo, MD, Department of Radiology and Research Institute of Radiology, University of Ulsan College of Medicine, Asan Medical Center, 88 Olympic-ro 43-gil, Songpa-gu, Seoul 05505, Korea.

- Tel: (822) 3010-4400• Fax: (822) 476-4719•E-mail: seojb@amc.seoul.kr

This is an Open Access article distributed under the terms of the Creative Commons Attribution Non-Commercial License (https:// creativecommons.org/licenses/by-nc/4.0) which permits unrestricted non-commercial use, distribution, and reproduction in any medium, provided the original work is properly cited. 
appropriate for the lung, a smooth kernel is better for the mediastinum. Because kernel selection affects quantitative analysis (2-4), CT images with different reconstruction kernels would be necessary for various diagnostic or quantitative purposes.

Although raw $\mathrm{CT}$ data are required to reconstruct $\mathrm{CT}$ images with different kernels, the data are usually not stored owing to their large file sizes and space limitations. On the other hand, multiple reconstructions with different kernels for all cases will increase the number of images that are generated, transmitted, and stored. These limitations in CT reconstruction have led to concerns regarding the comparability of quantitative results among different reconstruction kernels (2), and this problem is particularly evident in retrospective or longitudinal studies that cannot control technical parameters. Therefore, attempts to develop post-processing techniques that permit interconversion among $\mathrm{CT}$ images obtained with different kernels have been made (5-8). Gallardo-Estrella et al. (9) demonstrated that normalization of $\mathrm{CT}$ data reconstructed with different kernels reduces variation in emphysema quantification. However, the emphysema index (EI), which is derived from CT data reconstructed using the reference kernel (Siemens b31f), was altered after normalization, implying that the normalized EI does not exactly correspond to the EI obtained in original CT.

Kim et al. (10) recently demonstrated that convolutional neural networks (CNNs) can be taught differences between high- and low-resolution images (residual images) and that CNNs can be used to accurately and rapidly convert low-resolution images to high-resolution images. We hypothesized that this is also applicable for interconverting CT images obtained using different kernels because CT images between different kernels share most information. As EI is an established and widely accepted predictor for survival and acute exacerbation $(11,12)$ as well as disease status in patients with chronic obstructive lung disease (13), it was selected as a performance measure after kernel conversion in our study. Thus, the purpose of this study was to develop and validate a CNN architecture to convert CT images reconstructed with one kernel to images with different reconstruction kernels without using a sinogram.

\section{MATERIALS AND METHODS}

This retrospective study was approved by the Institutional Review Board, which waived the requirement for informed consent from patients (approval number: 2017-0951).

\section{Datasets}

The first dataset included CT images from 10 patients (five men and five women; mean age, $63.0 \pm 8.6$ years), obtained using Somatom Definition Edge (Siemens Healthineers, Forchheim, Germany) in May 2016. This dataset was initially used for developing an optimal protocol for quantification in patients with chronic obstructive pulmonary disease. CT images were reconstructed using the B10f, B30f, B50f, and B70f kernels, from smooth to sharp, according to the Siemens naming convention. This dataset was used to develop a kernel conversion algorithm and to evaluate its performance (referred to as Dataset 1 ).

The second dataset consisted of 300 CT scans obtained using Somatom Definition Edge from April to June 2017. The second dataset included two series of CT images reconstructed with the B30f and B50f kernels for the mediastinum and lung, respectively. We randomly selected 20 examinations for training another CNN model with an architecture identical to that of the model in Dataset 1. To validate this model for clinical quantitative use, we used $\mathrm{EI}$, and 30 chest $\mathrm{CT}$ scans among the remaining $\mathrm{CT}$ images in Dataset 2 that showed emphysema on visual assessment were selected by a chest radiologist.

\section{Development of CNN Architecture}

The proposed CNN architecture is shown in Figure 1 and consists of six convolutional layers with a $3 \times 3$ kernel size. Each layer, except the last one, has 64 filter banks that enabled the extraction of 64 distinctive feature maps. To maintain original resolution throughout the network, we excluded a pooling layer in the CNN structure. A rectified linear unit was used as an activation function at the end of each convolutional layer. As we applied the concept of residuals, the proposed CNN was trained to learn the difference between the target and input images.

Data preprocessing involved deconstruction of threedimensional $\mathrm{CT}$ volumes to multiple two-dimensional images. We used the original matrix size of $512 \times 512$ for both input and output images. For each distinct reconstruction kernel pair, we also generated a residual image by subtracting the target image from the input image.

The prepared image database was fed into the deep learning processing server with the Linux operating system (Ubuntu 14.04; Canonical, London, England) along with the Caffe deep learning framework (http://caffe.berkeleyvision. 


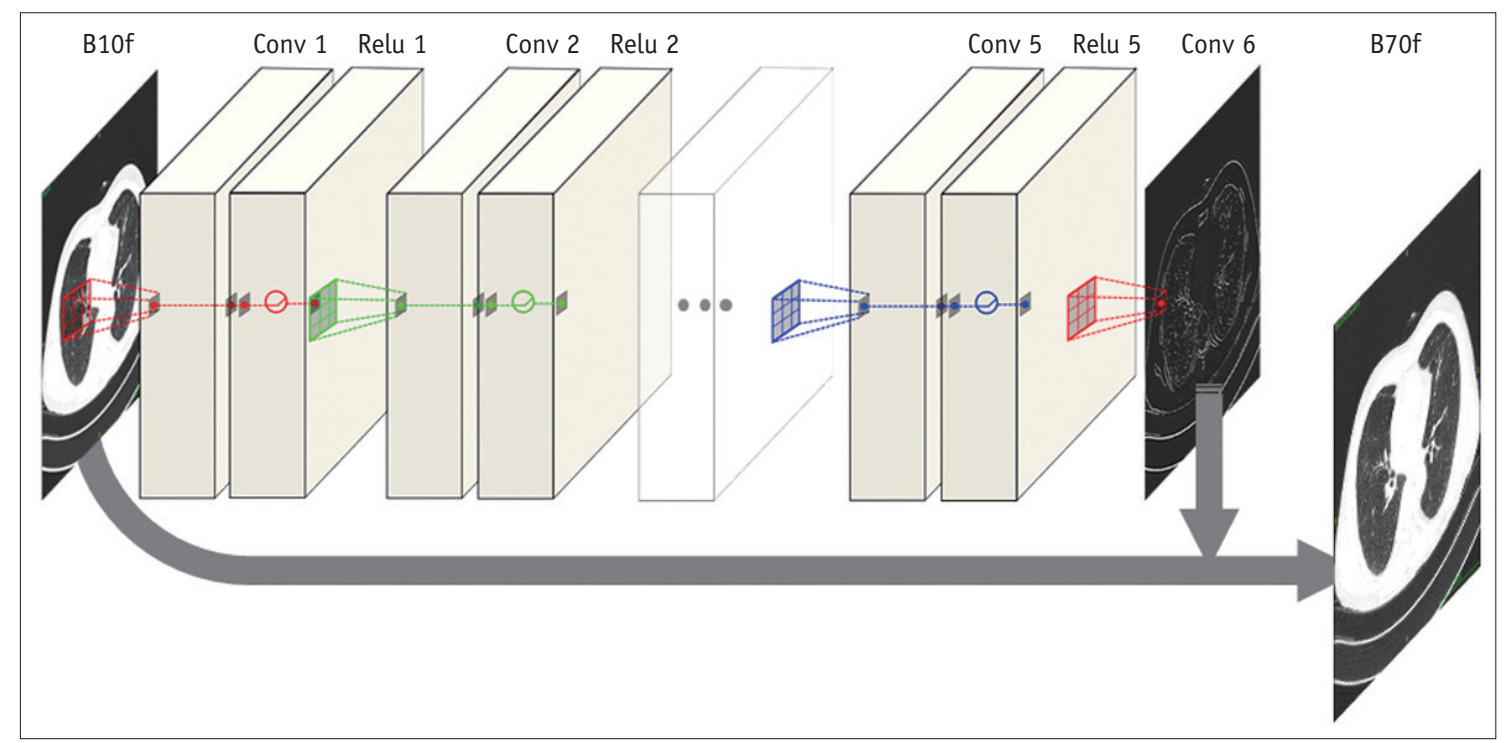

Fig. 1. Architecture of CNN. Proposed CNN architecture consists of six convolutional layers, each with $3 \times 3$ kernel with 64 filter banks. To maintain original resolution throughout network, we excluded pooling layer in CNN structure and used original matrix size of $512 \times 512$ for both input and output images. Rectified linear unit was used as activation function at end of each convolutional layer. As we applied concept of residuals, proposed CNN was trained to learn difference between target and input images, and final image was obtained by adding residual image (output) to input. $\mathrm{CNN}=$ convolutional neural network

org; Berkeley Vision and Learning Center, Berkeley, CA, USA) and CUDA 8.0/cuDNN 5.1 dependencies (Nvidia Corporation, Santa Clara, CA, USA) for graphics processing unit acceleration. The computing server had the Intel Xeon E52600 processor (Intel, Santa Clara, CA, USA), 64-GB RAM, and an Nvidia Titan X and two GeForce GTX 1080 graphics processing units.

We divided Dataset 1 into six, two, and two cases for training, validation, and testing, respectively. The six training image sets included 1990 images, and the validation set contained 679 images. Operational parameters for training are presented in Table 1.

Dataset 1 contains twelve distinctive conversion pairs and we developed 12 CNN models, corresponding to each of the 12 pairs, to obtain images with the desired CT reconstruction kernels from the given image(s). For Dataset 2 , separate CNN models were prepared using the $[\mathrm{B} 30 \mathrm{f} \rightarrow$ B50f] and [B50f $\rightarrow$ B30f] kernels.

\section{Testing CNN Architecture}

Two image sets (Test 1 and Test 2) from Dataset 1 that included $631 \mathrm{CT}$ images were used to test the performance of the proposed CNN. We calculated the root mean square error (RMSE) values for each conversion pair as a quantitative measurement using the following formula:
Table 1. Operational Parameters for Training Proposed Convolutional Neural Network-Based Model

\begin{tabular}{ll}
\hline \multicolumn{1}{c}{ Operational Parameters } & Values or Names \\
\hline Number of iteration (epoch) & 300 \\
Mini-batch sample size & 5 \\
Loss function & Sum of squares \\
Optimization method & ADAM (4) \\
Learning rate & 0.0001 \\
\hline ADAM = adaptive moment estimation & \\
RMSE $=\sqrt{\sum \frac{\left(y_{g}-y_{g t}\right)^{2}}{N}}$ &
\end{tabular}

Where, $y_{g}$ is the voxel value of the CT volume generated using the proposed CNN and $\mathrm{y}_{\mathrm{gt}}$ is the voxel value of the target CT volume.

In Dataset 2, we quantified the EI on CT images reconstructed with B30f, B50f, converted B30f, and converted B50f using a commercial software (Aview, Coreline Soft, Seoul, Korea) as well as RMSE. The quantified EIs on B50f and converted B30f images were compared with the EI from B30f images, because quantification of EI on CT images acquired using B30f is a widely acceptable approach (14).

\section{СT Acquisition}

CT images were acquired at full inspiration in the supine position without contrast enhancement, and the acquisition protocol was as follows: $120 \mathrm{kVp} ; 100-200 \mathrm{mAs} ; 1-\mathrm{mm}$ slice thickness; $1-\mathrm{mm}$ intervals; and collimation of $0.75 \mathrm{~mm}$. 


\section{Statistical Analysis}

All values are expressed as mean \pm standard deviation.

For continuous variables, the normality of distribution was assessed using the Kolmogorov-Smirnov test. In Dataset 2, Bland-Altman plots were used to assess differences between EI with B30f and that with other reconstruction kernels (15). EI comparisons among different kernels were performed using the Wilcoxon signed-rank test. All statistical analyses were performed using MedCalc, v.17.8 (MedCalc Software, Mariakerke, Belgium). $P$ values of $<0.05$ were considered significant.

\section{RESULTS}

\section{Performance in Dataset 1}

Performance test results of the proposed CNNs for each conversion pair in the two image sets are shown in Tables 2 and 3, respectively. The mean reduction in RMSE for the converted images was $64.4 \pm 16.1 \%$ (range, $29.5-79.4 \%$ ) in Test 1 and $67.0 \pm 16.1 \%$ (range, $31.5-82.2 \%$ ) in Test 2. In pooled data for Tests 1 and 2, the converted images showed a substantial reduction in RMSE values (mean reduction, $65.7 \pm 15.8 \%$ ) for all conversion pairs except images converted from B10f to B70f in Test 1, where the decrease

Table 2. RMSE Values of Subtracted CT Volumes in Test 1

\begin{tabular}{lccc}
\hline \multicolumn{1}{c}{ Conversion Pairs (Input, Target Image) } & Original (HU) & Converted (HU) & Differences (\%) \\
\hline From smooth to sharp kernel & & & \\
B10f, B30f & $16.31 \pm 2.04$ & $33.08 \pm 8.71$ & $-63.2 \pm 2.9$ \\
B10f, B50f & $58.53 \pm 11.02$ & $84.18 \pm 25.54$ & $-44.1 \pm 5.2$ \\
\hline B10f, B70f & $119.48 \pm 28.10$ & $18.29 \pm 4.34$ & $-30.5 \pm 5.4$ \\
\hline B30f, B50f & $44.57 \pm 9.64$ & $52.95 \pm 15.35$ & $-59.1 \pm 1.8$ \\
B30f, B70f & $107.47 \pm 27.29$ & $22.82 \pm 6.63$ & $-51.1 \pm 2.0$ \\
\hline B50f, B70f & $66.03 \pm 18.32$ & & $-65.5 \pm 1.9$ \\
From sharp to smooth kernel & & $4.05 \pm 0.36$ & $-75.0 \pm 1.7$ \\
\hline B30f, B10f & $16.31 \pm 2.04$ & $12.91 \pm 2.65$ & $-77.9 \pm 1.8$ \\
\hline B50f, B10f & $58.53 \pm 11.02$ & $10.72 \pm 2.37$ & $-75.8 \pm 2.1$ \\
\hline B50f, B30f & $44.57 \pm 9.64$ & $24.89 \pm 6.55$ & $-79.1 \pm 2.4$ \\
\hline B70f, B10f & $119.48 \pm 28.10$ & $22.14 \pm 5.98$ & $-79.2 \pm 2.6$ \\
\hline B70f, B30f & $107.47 \pm 27.29$ & $16.95 \pm 5.03$ & $-74.2 \pm 2.4$ \\
\hline B70f, B50f & $66.03 \pm 18.32$ & & \\
\hline
\end{tabular}

Data are presented as mean \pm SD (HU). Original: RMSE by subtracting between input and target images. Converted: RMSE by subtracting converted images from target images. Differences: $100 \times$ (converted RMSE - original RMSE) / original RMSE. HU = Hounsfield unit, RMSE = root mean square error, $\mathrm{SD}=$ standard deviation

Table 3. RMSE Values of Subtracted CT Volumes in Test 2

\begin{tabular}{|c|c|c|c|}
\hline Conversion Pairs (Input, Target Image) & Original & Converted & Differences $(\%)$ \\
\hline \multicolumn{4}{|l|}{ From smooth to sharp kernel } \\
\hline B10f, B30f & $15.02 \pm 0.87$ & $5.17 \pm 0.45$ & $-65.6 \pm 2.3$ \\
\hline B10f, B50f & $53.50 \pm 5.08$ & $28.84 \pm 4.52$ & $-46.4 \pm 4.0$ \\
\hline B10f, B70f & $109.50 \pm 14.07$ & $74.97 \pm 13.46$ & $-31.9 \pm 4.0$ \\
\hline B30f, B50f & $40.68 \pm 4.65$ & $15.72 \pm 2.15$ & $-61.4 \pm 1.5$ \\
\hline B30f, B70f & $98.50 \pm 13.94$ & $45.49 \pm 7.47$ & $-53.9 \pm 1.5$ \\
\hline B50f, B70f & $60.63 \pm 9.56$ & $18.77 \pm 2.70$ & $-69.0 \pm 0.7$ \\
\hline \multicolumn{4}{|l|}{ From sharp to smooth kernel } \\
\hline B30f, B10f & $15.02 \pm 0.87$ & $3.44 \pm 0.18$ & $-77.1 \pm 0.8$ \\
\hline B50f, B10f & $53.50 \pm 5.08$ & $10.85 \pm 1.16$ & $-79.7 \pm 1.1$ \\
\hline B50f, B30f & $40.68 \pm 4.65$ & $8.81 \pm 1.10$ & $-78.3 \pm 1.3$ \\
\hline B70f, B10f & $109.50 \pm 14.07$ & $19.92 \pm 2.15$ & $-81.7 \pm 1.3$ \\
\hline B70f, B30f & $98.50 \pm 13.94$ & $17.55 \pm 1.56$ & $-82.0 \pm 1.5$ \\
\hline B70f, B50f & $60.63 \pm 9.56$ & $13.63 \pm 1.63$ & $-77.3 \pm 1.3$ \\
\hline
\end{tabular}

Data are presented as mean \pm SD (HU). Original: RMSE by subtracting between input and target images. Converted: RMSE by subtracting converted images from target images. Differences: $100 \times$ (converted RMSE - original RMSE) / original RMSE 


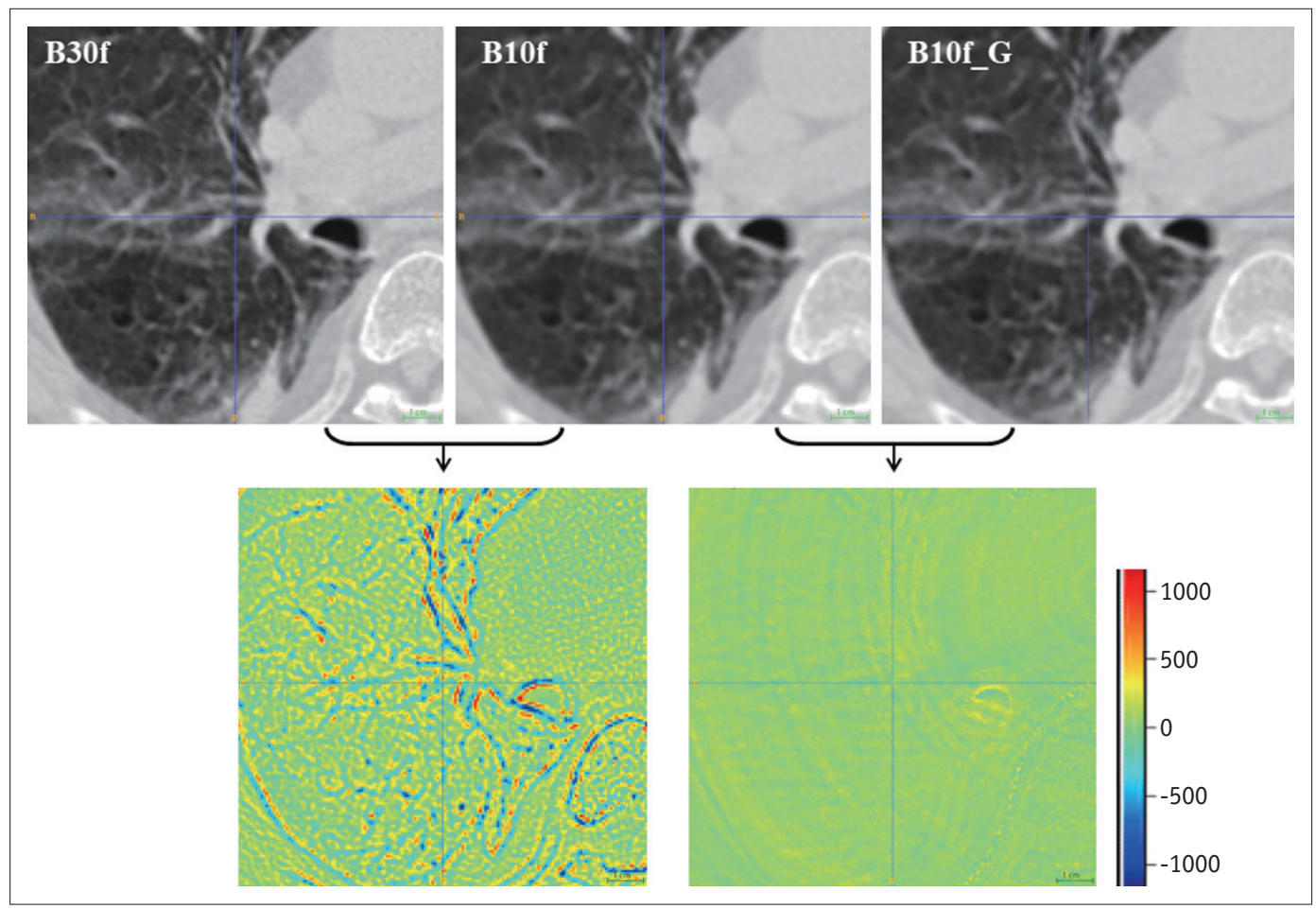

A

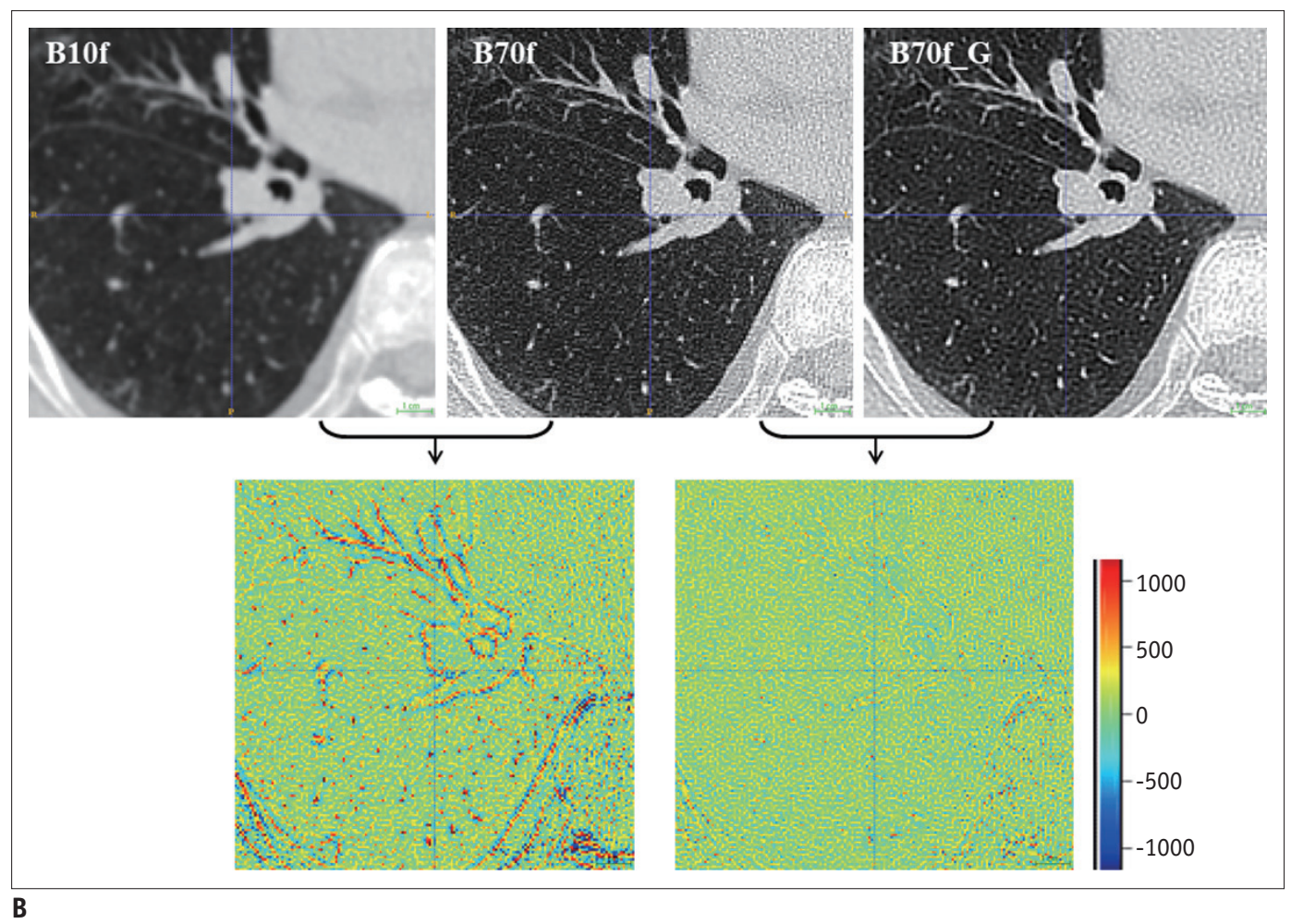

Fig. 2. Comparisons of original and converted CT images using CNN. Input image, ground-truth image, and converted image are presented on upper panel, and difference images are located on bottom panel. In difference image, green indicates zero, blue represents negative values, and red indicates positive values.

A. Pronounced differences between B10f and B30f images mostly disappeared after applying proposed conversion scheme (RMSE from 16.95 HU to 3.12 HU). B. Similar results were observed in kernel conversion from B10f to B70f (RMSE from 149.06 HU to 99.21 HU). However, speckled error regions remained owing to difficulties in kernel conversion from B10f to B70f and high signal-to-noise ratio in B70f. HU $=$ Hounsfield unit, RMSE $=$ root mean square error 
in RMSE values was much lower at $29.5 \%$ compared with those in the original images.

Our model demonstrated good conversion ability from smooth to sharp kernels, with a mean RMSE reduction of $51.9 \%$ in Test 1 and $54.6 \%$ in Test 2, and from sharp to smooth kernels, with a mean RMSE reduction of $77.0 \%$ in Test 1 and $79.4 \%$ in Test 2.

Among the kernel conversion pairs used, B30f to B10f in Test 2 yielded the lowest RMSE ( $3.44 \pm 0.18$ Hounsfield unit [HU]), whereas B10f to B70f in Test 1 showed the highest RMSE $(84.18 \pm 25.5 \mathrm{HU})$. RMSE values tended to increase during conversion from smooth to sharp kernels and between much different kernels (Fig. 2). For all conversion pairs, RMSE values were lower in Test 2 than in Test 1 . This CNN could rapidly generate conversion results at a rate of $0.065 \mathrm{~s} /$ slice and took approximately 20 seconds for converting one entire volume.
B50f) and (converted B50f, B50f) and - $78.7 \pm 1.8 \%$ between (B50f, B30f) and (converted B30f, B30f).

The mean EIs from B30f, B50f, converted B30f, and converted B50f images were $5.4 \pm 7.2 \%, 15.3 \pm 7.2 \%, 5.9 \pm$ $7.3 \%$, and $16.8 \pm 7.5 \%$, respectively (Fig. 3 ). KolmogorovSmirnov test revealed that the EI distributions in B30f and converted B30f images were not normal $(p=0.003$ and $p=$ 0.012 , respectively), whereas those in B50f and converted B50f images were normal ( $p=0.059$ and $p=0.090$, respectively). Comparisons between B30f and other kernels, namely B50f, converted B30f, and converted B50f, showed significant differences $(p<0.001, p=0.001$, and $p<0.001$, respectively).

Bland-Altman plots showed that the $95 \%$ limits of agreement between B30f and other kernels (B50f and converted B30f) ranged from $-14.1 \%$ to $-2.6 \%$ (mean, $-8.3 \%$ ) and from $-2.3 \%$ to $0.7 \%$ (mean, $-0.8 \%$ ), respectively (Fig. 4 ).

\section{Performance in Dataset 2}

The reduction in RMSE was $-59.6 \pm 5.5 \%$ between (B30f,
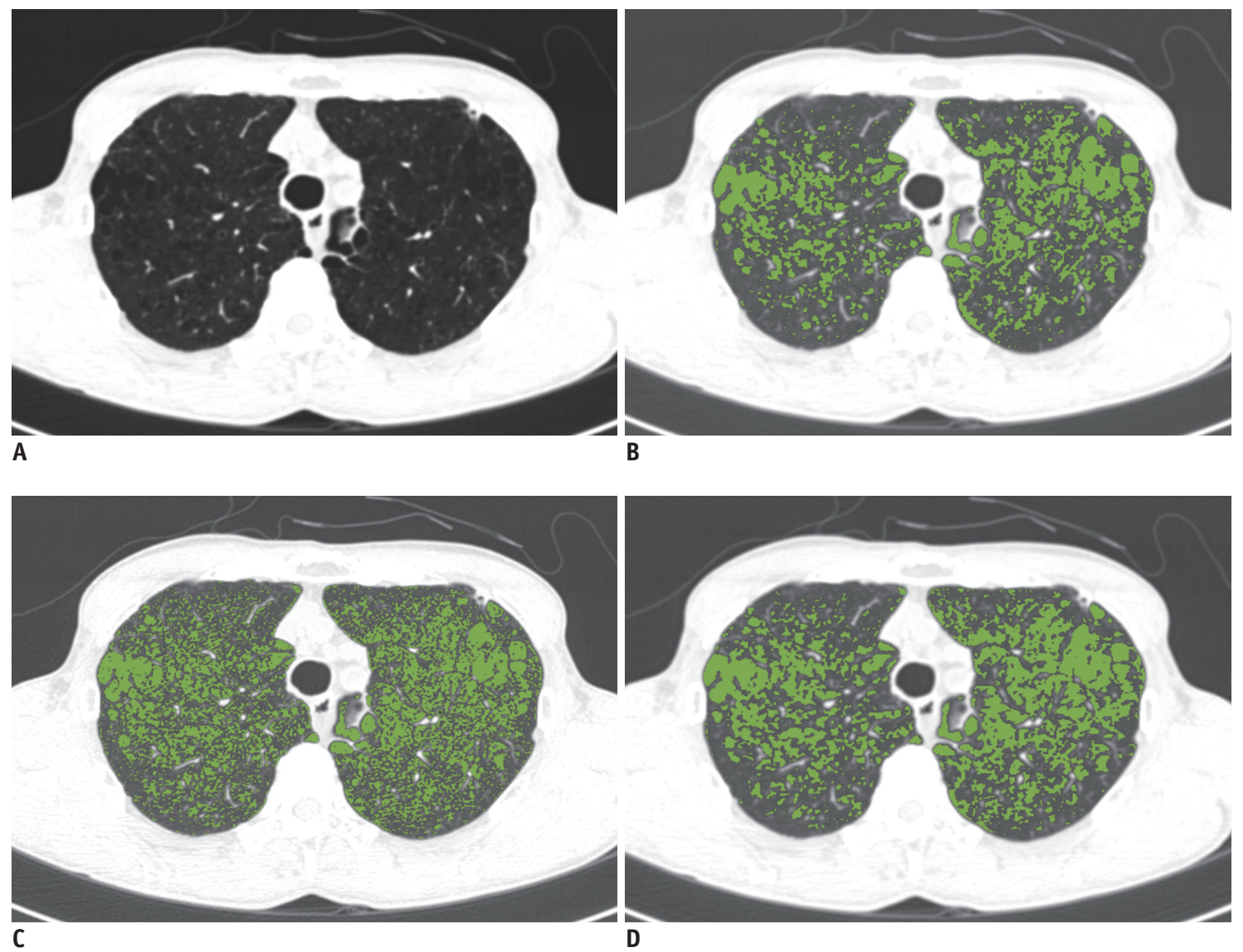

Fig. 3. Influence of kernels on EI.

EI was calculated from CT images reconstructed using different kernels in 72-year-old male [(A): B30f]. Results for EI were 9.9\% in B30f kernel image (B), 20.2\% in B50f kernel image (C), and 12.1\% in converted B30f kernel image (D). EI = emphysema index 




Fig. 4. Bland-Altman plots showing association between B30f and other kernels (B50f and converted B30f).

A, B. Graphs showing differences between B30f and other kernels (B50f and converted B30f). X-axis represents EI in B30f, and Y-axis represents differences in EI between B30f and other kernels. (A) B30f and B50f and (B) B30f and converted B30f. 95\% limits of agreements ranged from $-14.1 \%$ to $-2.6 \%$ (mean, $-8.3 \%$ ) and from $-2.3 \%$ to $0.7 \%$ (mean, $-0.8 \%$ ), respectively. $\mathrm{SD}=$ standard deviation

\section{DISCUSSION}

In our study, we described a new CNN method to convert $\mathrm{CT}$ images from different kernels and demonstrated an adequate model performance during clinical use by comparing EI quantifications between original and converted images.

The necessity of $\mathrm{CT}$ image conversion among different reconstruction kernels has remained consistent, because controlling the protocols used for image acquisition is not always possible. Several studies have attempted to develop a convenient and universal method for image conversion among different reconstruction kernels. Schaller et al. (1) introduced a method to simulate smoother kernels by using a Gaussian filter to approximate the ratio between smooth and sharp kernels. Ohkubo et al. (8) reported an advanced filtering method to create $\mathrm{CT}$ images with different kernels. However, these studies did not evaluate the suitability of the converted images for quantification, and these methods require additional CT scans for calculating conversion parameters such as the point-spread function. GallardoEstrella et al. (9) demonstrated that normalization of CT data reconstructed using different kernels reduced variation during emphysema quantification. They used energy coefficients, which were obtained by analyzing the energy in different frequency bands in scans with the reference kernel. Although the method did not require additional $\mathrm{CT}$, it is difficult to directly predict normalized EI even in reference images.

Our proposed CNN-based method of kernel conversion has several advantages over the other published methods described above $(5,8,9)$. First, our model generated converted $\mathrm{CT}$ images from any reconstruction kernel using an identical architecture. We presented data from 12 pairs of kernel conversions using the same CNN model and showed that changing the input data according to a desired kernel alone was sufficient for obtaining target images, making our proposed CNN-based model easily adaptable and expandable. The method used here could feasibly be adapted to other CT applications such as noise reduction in low-dose CT and vendor-based differences in images (16).

Second, our model uses the original CT resolution (512 x 512) throughout the conversion process with an end-toend way. Many other $\mathrm{CNN}$-based studies used pre-trained networks originally trained using data from ImageNet, and it was necessary to reduce the image size to $256 \times 256$ and lower 12-bit $\mathrm{HU}$ values to 8 -bit red, green, and blue values. However, we aimed to use the original size of CT images and the complete range of attenuation values (HU). To achieve these goals, we devised a simpler CNN model and decomposed the three-dimensional scans into twodimensional slices to compensate for limitations owing to small dataset size. This scheme was performed in the end-to-end manner and required no additional calculation of parameters. This can enhance the robustness of the proposed scheme. 


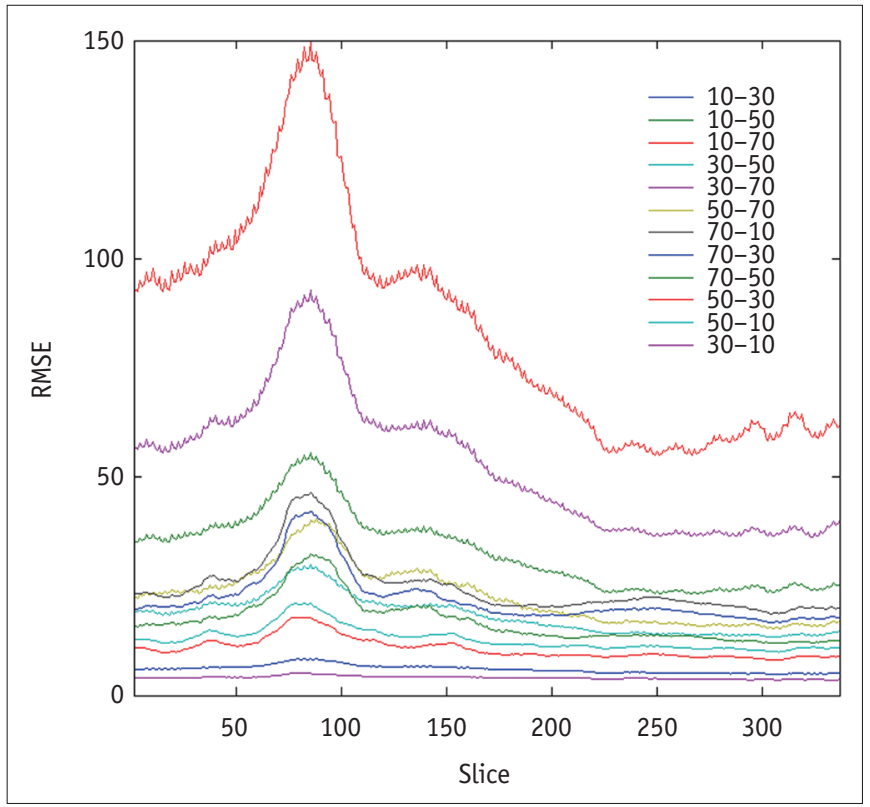

A

Fig. 5. Changes in RMSE along $z$ axis.

A, B. Graphs showing differences in RMSE between original image and converted images along $z$ axis in Test 1 and Test 2. There was trend of increase from upper to lower thorax.

Third, we incorporated residual learning in the model, i.e., for converting B10f to B70f images, B10f images are input to a specific CNN model and residual images are generated as the output. The final image is obtained by adding residual images to the input. This scheme improved performance and reduced the training time of our CNN models because the training time and RMSE values for conversion from B10f to B70f were 4.5 hours and $75.0 \mathrm{HU}$, respectively, with 300 iterations. However, without residual learning, the performance was $>3$ times lower (RMSE, 250.7 $\mathrm{HU})$, and we could not achieve comparable results even with a 10 -fold increase in training time. These observations attest to the effectiveness of residual learning in the proposed method.

Interestingly, there were differences in the RMSE along the $z$ direction (Fig. 5). We found a trend of an increase from the upper to lower thorax. This might be caused by several reasons. First, the upper part is more stable than the lower part from breathing. Second, the spatial variability of abdominal organs could make the training the CNN models more difficult.

Kernel conversion from B50f to B30f showed considerably reduced agreement for EI quantification. Gallardo-Estrella et al. (9) revealed that bias and limits of agreement decreased from $7.7 \%(2.4 \%, 12.9 \%)$ to $0.3 \%(-1.0 \%, 1.5 \%)$ after normalization of B $45 \mathrm{f}$ to B31f. Although we reported slightly wider agreement values compared with GallardoEstrella et al. (9), we believe that this could be attributed to differences in the study population and reconstruction kernel used between two studies. In addition to the advantages mentioned above, we believe that our method would substantially improve along with advances in deep learning techniques.

Nonetheless, our study has several limitations. First, only a small sample was included in our study. As each image was used for CNN development, validation, and testing, we believe that the sample was sufficient in this regard. In EI measurement, our study population did not represent the entire spectrum of patients with varying degrees of emphysema, owing to the rarity of patients with severe emphysema. However, Bland-Altman plots demonstrated that measurement differences showed no association with the extent of emphysema. Next, CT quantification was performed using only one CT protocol and CT scanner type. Therefore, an external validation under different conditions is necessary to test the wide applicability of our CNN model.

In conclusion, CT kernel conversion based on CNNs showed adequate performance with high accuracy and speed and offered potential for clinical applications.

\section{Conflicts of Interest}

The authors have no financial conflicts of interest. 
ORCID

Joon Beom Seo

https://orcid.org/0000-0003-0271-7884

Sang Min Lee

https://orcid.org/0000-0001-7627-2000

June-Goo Lee

https://orcid.org/0000-0002-1380-6682

\section{REFERENCES}

1. Schaller S, Wildberger JE, Raupach R, Niethammer M, Klingenbeck-Regn K, Flohr T. Spatial domain filtering for fast modification of the tradeoff between image sharpness and pixel noise in computed tomography. IEEE Trans Med Imaging 2003;22:846-853

2. Boedeker KL, McNitt-Gray MF, Rogers SR, Truong DA, Brown MS, Gjertson DW, et al. Emphysema: effect of reconstruction algorithm on CT imaging measures. Radiology 2004;232:295301

3. Behrendt FF, Das M, Mahnken AH, Kraus T, Bakai A, Stanzel S, et al. Computer-aided measurements of pulmonary emphysema in chest multidetector-row spiral computed tomography: effect of image reconstruction parameters. J Comput Assist Tomogr 2008;32:899-904

4. Wang $Y$, de Bock GH, van Klaveren RJ, van Ooyen P, Tukker W, Zhao $Y$, et al. Volumetric measurement of pulmonary nodules at low-dose chest CT: effect of reconstruction setting on measurement variability. Eur Radiol 2010;20:1180-1187

5. Weiss KL, Cornelius RS, Greeley AL, Sun D, Chang IY, Boyce W0, et al. Hybrid convolution kernel: optimized CT of the head, neck, and spine. AJR Am J Roentgenol 2011;196:403406

6. Wildberger JE, Mahnken AH, Flohr T, Raupach R, Weiss C, Günther RW, et al. Spatial domain image filtering in computed tomography: feasibility study in pulmonary embolism. Eur Radiol 2003;13:717-723
7. Lapp RM, Kyriakou Y, Kachelriess M, Wilharm S, Kalender WA. Interactively variable isotropic resolution in computed tomography. Phys Med Biol 2008;53:2693-2713

8. Ohkubo M, Wada S, Kayugawa A, Matsumoto T, Murao K. Image filtering as an alternative to the application of a different reconstruction kernel in CT imaging: feasibility study in lung cancer screening. Med Phys 2011;38:3915-3923

9. Gallardo-Estrella L, Lynch DA, Prokop M, Stinson D, Zach J, Judy PF, et al. Normalizing computed tomography data reconstructed with different filter kernels: effect on emphysema quantification. Eur Radiol 2016;26:478-486

10. Kim J, Lee JK, Lee KM. Accurate image super-resolution using very deep convolutional networks (p. 1646-1654). 2016 The IEEE Conference on Computer Vision and Pattern Recognition (CVPR); 2016 June 27-30;Las Vegas, NV, USA

11. Haruna A, Muro S, Nakano Y, Ohara T, Hoshino Y, Ogawa E, et al. CT scan findings of emphysema predict mortality in COPD. Chest 2010;138:635-640

12. Han MK, Kazerooni EA, Lynch DA, Liu LX, Murray S, Curtis JL, et al.; COPDGene Investigators. Chronic obstructive pulmonary disease exacerbations in the COPDGene study: associated radiologic phenotypes. Radiology 2011;261:274-282

13. Lee YK, Oh YM, Lee JH, Kim EK, Lee JH, Kim N, et al.; KOLD Study Group. Quantitative assessment of emphysema, air trapping, and airway thickening on computed tomography. Lung 2008;186:157-165

14. Schroeder JD, McKenzie AS, Zach JA, Wilson CG, CurranEverett D, Stinson DS, et al. Relationships between airflow obstruction and quantitative CT measurements of emphysema, air trapping, and airways in subjects with and without chronic obstructive pulmonary disease. AJR Am J Roentgenol 2013;201:W460-W470

15. Bland JM, Altman DG. Statistical methods for assessing agreement between two methods of clinical measurement. Lancet 1986;1:307-310

16. Lee JG, Jun S, Cho YW, Lee H, Kim GB, Seo JB, et al. Deep learning in medical imaging: general overview. Korean $\mathrm{J}$ Radiol 2017;18:570-584 